Readers are invited to submit letters for publication in this department. Submit letters online at http://joem.edmgr.com. Choose "Submit New Manuscript." A signed copyright assignment and financial disclosure form must be submitted with the letter. Form available at www.joem.org under Author and Reviewer information.

\section{Anti-SARS-CoV-2 IgA Response in Baseline Seronegative and Seropositive Recipients of BNT162b2 mRNA COVID-19 Vaccine}

\section{To the Editor:}

W e read with interest the article by Iddins et al., ${ }^{1}$ reporting the immune response anti-SARS-CoV-2 IgG against the receptor binding domain (RBD) spike protein in healthcare workers. Since the risk of infection and poor outcomes seem also largely dependent on efficient anti-SARSCoV-2 IgA response, assessing this antibody class is pivotal. ${ }^{2}$

Thus, we analyzed serum levels of total anti-spike RBD Ig, (Elecsys Anti-
ELISA; Euroimmun, Lübeck, Germany), in 24 healthcare workers undergoing vaccination with BNT162b2 mRNA COVID-19 at Peschiera del Garda hospital (Italy). This cohort comprised two matched samples of 12 baseline SARS-CoV-2 seronegative subjects (median age 40 years, interquartile range 31-52 years; 6 women) and 12 baseline SARS-CoV-2 seropositive subjects (median age 41 years, interquartile range $31-52$ years; 6 women). Serum samples were drawn before the first vaccine dose, at 21 days (before the second vaccine dose) and 50 days afterwards. The study was conducted in accordance with the Helsinki Declaration and cleared by the Ethics Committee of Verona and Rovigo Provinces (3246CESC).

The results are summarized in Table 1. Levels of all antibodies increased in both baseline SASR-CoV-2 seronegative and seropositive cohorts, though such increase continued after the second dose in baseline seronegative subjects, while reached a plateau in baseline seropositive subjects. In agreement with earlier data published by Vicenti et $\mathrm{al}^{3}{ }^{3}$ anti-SARS-
Our results, reporting anti-SARSCoV-2 IgA antibodies response after BNT162b2 mRNA COVID-19 vaccination, suggest that consideration could be given to delay and/or omit the second vaccine dose in baseline SARS-CoV-2 seropositive subjects.

Gian Luca Salvagno, MD Section of Clinical Biochemistry University of Veona Verona, Italy Sevice of Laboratory Medicine Pederzoli Hspital

Peschiera del Garda, Italy

Brandon M. Henry, MD The Heart Institute, Cincinnati Children's Hospital Medical Center Cincinnati, $\mathrm{OH}$

Giuseppe Lippi, MD Section of Clinical Biochemistry University of Verona Verona, Italy

TABLE 1. Serum Levels of anti-SARS-CoV-2 Antibodies (Median and Interquartile Range) in Baseline SARS-CoV-2 Seronegative and Seropositive Healthcare Workers, Undergoing BNT162b2 mRNA COVID-19 Vaccination

\begin{tabular}{|c|c|c|c|c|c|c|c|c|c|}
\hline \multirow[b]{2}{*}{ Antibodies } & \multicolumn{2}{|c|}{ Baseline } & \multirow[b]{2}{*}{$\boldsymbol{P}^{*}$} & \multicolumn{2}{|c|}{21 days } & \multirow[b]{2}{*}{$\boldsymbol{P}^{*}$} & \multicolumn{2}{|c|}{50 days } & \multirow[b]{2}{*}{$P^{*}$} \\
\hline & Seronegative & Seropositive & & Seronegative & Seropositive & & Seronegative & Seropositive & \\
\hline $\begin{array}{c}\text { Anti-SARS-CoV-2 RBD } \\
\text { Total Ig (AU/mL) }\end{array}$ & $\begin{array}{c}0.4 \\
(0.4-0.4)\end{array}$ & $\begin{array}{c}92.5 \\
(15.9-141.5)\end{array}$ & $<0.001$ & $\begin{array}{c}57.2 \\
(21.2-98.0)\end{array}$ & $\begin{array}{c}20,585.5 \\
(11,902.0-24,527.5)\end{array}$ & $<0.001$ & $\begin{array}{c}1629.0 \\
(1136.3-2587.0)(\end{array}$ & $\begin{array}{c}19,984.0 \\
14,394.8-25,000.0)\end{array}$ & $<0.001$ \\
\hline $\begin{array}{l}\text { Anti-SARS-CoV-2 RBD } \\
\operatorname{IgG}(\mathrm{AU} / \mathrm{mL})\end{array}$ & $\begin{array}{c}0.2 \\
(0.1-0.2)\end{array}$ & $\begin{array}{c}10.0 \\
(8.0-14.4)\end{array}$ & $<0.001$ & $\begin{array}{c}41.7 \\
(23.1-88.1)\end{array}$ & $\begin{array}{c}340.4 \\
(241.8-433.2)\end{array}$ & $<0.001$ & $\begin{array}{c}341.2 \\
(263.7-641.2)\end{array}$ & $\begin{array}{c}306.4 \\
(262.7-358.2)\end{array}$ & 0.068 \\
\hline $\begin{array}{l}\text { Anti-SARS-CoV-2 S1 } \\
\text { IgA (ratio) }\end{array}$ & $0.23(0.16-0.31$ & $1.17(0.78-1.70)$ & $<0.0013$ & $3.15(2.02-5.65)$ & $8.97(8.79-10.41)$ & $<0.0015$ & $5.76(3.67-7.28)$ & $8.76(8.01-9.75)$ & $<0.001$ \\
\hline
\end{tabular}

SARS-CoV-2S immunoassay on Roche Cobas 6000; Roche Diagnostics, Basel, Switzerland), anti-spike RBD IgG (ACCESS SARS-CoV-2 IgG II on Access 2; Beckman Coulter, Brea CA), and anti-spike $\mathrm{S} 1$ subunit IgA (Anti-SARS-CoV-2 ELISA IgA, manual

Funding source: None.

Conflicts of interest: The authors declare that they have no conflicts of interest.

Address correspondence to: Giuseppe Lippi, MD, Section of Clinical Biochemistry, University Hospital of Verona, Piazzale L.A. Scuro, 10, 37134 Verona, Italy (giuseppe.lippi@univr.it).

Copyright (C) 2021 American College of Occupational and Environmental Medicine

DOI: $10.1097 / \mathrm{JOM} .0000000000002362$
CoV-2 RBD IgG levels after the first dose in baseline seropositive subjects were comparable to those achieved after two doses in baseline seronegative subjects (340.4 vs. 341.2 AU $/ \mathrm{mL} ; P=0.259)$, while those of anti-SARS-CoV-2 RBD Total Ig (20,585.5 vs. $1629.0 \mathrm{AU} / \mathrm{mL} ; P<0.001)$ and antiSARS-CoV-2 S1 Ig A (8.97 vs. 5.76 ratio; $P<0.001)$ were significantly higher. After the second dose, levels of anti-SARS-CoV2 RBD Total Ig and Anti-SARS-CoV-2 S1 Ig A were still significantly higher in baseline seropositive subjects than in the seronegative cohort (both $P<0.001$ ), while anti-SARS-CoV-2 RBD IgG levels were comparable $(P=0.068)$.

\section{REFERENCES}

1. Iddins BO, Buck B, Cato T, et al. mRNA SARSCoV-2 immunization confers robust antibody response in occupational healthcare workers and fosters workplace safety. J Occup Environ Med. 2021;63:e314-e317.

2. Quinti I, Mortari EP, Fernandez Salinas A, et al. $\operatorname{Ig} \mathrm{A}$ antibodies and IgA deficiency in SARSCoV-2 infection. Front Cell Infect Microbiol. 2021;11:65589.

3. Vicenti I, Gatti F, Scaggiante R, et al. Singledose BNT162b2 mRNA COVID-19 vaccine significantly boosts neutralizing antibody response in health care workers recovering from asymptomatic or mild natural SARSCoV-2 infection. Int J Infect Dis. 2021;108: $176-178$. 